Running head: Attentional bias to pain-related information

\title{
Attentional bias to pain-related information: A meta-analysis of dot-probe studies
}

*Denotes shared first authorship

${ }^{a}$ School of Psychology; University of Sydney, Australia

${ }^{\mathrm{b}}$ Department of Experimental Psychology; Ghent University, Belgium

${ }^{c}$ Research Unit INSIDE, Institute for Health and Behaviour; Faculty of Language and

Literature, Humanities, Arts and Education; Luxembourg University, Luxembourg

\section{Author for correspondence:}

Jemma Todd, School of Psychology; University of Sydney, Sydney, NSW, 2006, Australia.

Email: jemma.todd@sydney.edu.au

\section{Contact details of other authors:}

Louise Sharpe: louise.sharpe@sydney.edu.au

Dimitri van Ryckeghem: dimitri.vanryckeghem@uni.lu

Geert Crombez: geert.crombez@ugent.be 
ATTENTIONAL BIAS TO PAIN-RELATED INFORMATION

\begin{abstract}
Studies investigating attentional biases towards pain information vary widely in both design and results. The aim of this meta-analysis was to determine the degree to which attentional biases towards pain occur when measured with the dot-probe task. A total of 2168 references were screened, resulting in a final sample of 4466 participants from 52 articles. Participants were grouped according to pain experience: chronic pain, acute pain, anticipating experimental/procedural pain, social concern for pain, or healthy people. In general, results revealed a significant, but small bias towards pain words $(d=0.136)$, and pain pictures $(d=$ 0.110) in chronic pain patients, but not in those with acute pain, those anticipating pain, or healthy people. Follow-up analyses revealed an attentional bias towards sensory pain words in the chronic pain group $(d=0.198)$, and the acute pain group $(d=0.303)$, but not other groups. In contrast, attentional biases towards affective pain stimuli were not significant for any pain groups. This meta-analysis found support for attentional biases towards sensory pain stimuli in patients with chronic pain in comparison to healthy individuals across a range of common parameters. Future researchers need to consider task design when seeking to optimally measure pain-relevant attentional biases.
\end{abstract}

Keywords: Pain; attentional bias; meta-analysis; dot-probe 
ATTENTIONAL BIAS TO PAIN-RELATED INFORMATION

\section{Introduction}

Chronic pain is highly prevalent, affecting approximately $20 \%$ of individuals globally, having serious effects on quality of life (Johannes, Le, Zhou, Johnston, \& Dworkin, 2010; Leadley, Armstrong, Lee, Allen, \& Kleijnen, 2012) and is associated with high rates of disability (Vos et al., 2012). Literature has emphasised the importance of taking a biopsycho-social approach to chronic pain, in which psychological and social factors interact with biomedical factors to bring about the experience of pain and associated illness and disability (Gatchel, Peng, Peters, Fuchs, \& Turk, 2007). There have been numerous theories of pain that are informed by a bio-psycho-social perspective, many of which share commonalities. For example, fear of pain and catastrophizing are often considered to lead to an interpretation of pain as threatening or potentially harmful (Crombez, Eccleston, Van Damme, Vlaeyen, \& Karoly, 2012; Todd et al., 2015; Vlaeyen \& Linton, 2000). This experience can in turn lead to increased attentional vigilance towards pain and pain-related cues (Todd et al., 2015), in order to solve the problem of pain (Aldrich, Eccleston, \& Crombez, 2000; Eccleston \& Crombez, 2007) or to avoid or reduce further pain (Sharp, 2001). The prioritisation of pain at the cost of other information can result in reduced activity and can contribute to poor mood and physical function, as well as increased disability, further maintaining the pain experience (Vlaeyen \& Linton, 2000; Vlaeyen, Morley, \& Crombez, 2016). As such, attention is a key feature in many models of pain, and in particular attentional biases towards pain information may be important.

The use of response time paradigms as an indirect measure to infer cognitive processes, such as attention, has a long tradition in psychology (Donders, 1969). Mental chronometry is still one of the core paradigms in cognitive and experimental psychology (Bundesen, 1990; Posner, 1980; Theeuwes, 2010). This is also the case for the investigation of attention bias. The emotional adaptation (Mathews \& MacLeod, 1985) of the original 
ATTENTIONAL BIAS TO PAIN-RELATED INFORMATION

Stroop (1935) task has been used to document to what extent words that were emotionally laden for patients with anxiety disorders interfered with the colour naming of these words. Participants were instructed to read aloud the print colours of displayed words, which were anxiety related or neutral, while ignoring the meaning of the words. It was found that the presentation of anxiety-related words interfered with responding, with longer latencies being indicative of more attention being paid to the emotional content of the word. In 1986 MacLeod, Mathews, and Tata (1986) introduced the dot probe task, which allowed researchers to infer to what extent patients were paying attention to emotionally laden symbolic stimuli (e.g. pictures and words). In the dot-probe task, emotionally laden and neutral stimuli are simultaneously presented at different locations of a display, after which one of the stimuli is replaced by a probe. The reaction time to respond to the location of the probe is measured. Attentional bias typically results in longer response latencies for probes that replace the neutral stimulus (incongruent trials) in comparison to those that replace the emotionally laden stimulus (congruent trials). Both tasks have been frequently used to study attentional bias in various situations, such as addiction (Field, Munafò, \& Franken, 2009), obesity (Hendrikse et al., 2015), eating disorders (Brooks, Prince, Stahl, Campbell, \& Treasure, 2011), phobia and anxiety disorders (Bar-Haim, Lamy, Pergamin, BakermansKranenburg, \& van Ijzendoorn, 2007), depression (Gotlib, Krasnoperova, Yue, \& Joormann, 2004), sleep-related disorders (Macmahon, Broomfield, \& Espie, 2006), chronic fatigue (Hou, Moss-Morris, Bradley, Peveler, \& Mogg, 2008), and also pain. In pain research, the emotionally laden stimuli have been replaced by pain-related stimuli to investigate biased attention for pain information (Asmundson, Kuperos, \& Ron Norton, 1997; Pearce \& Morley, 1989).

The existence of pain-related attentional biases has been supported by research, although findings are not always consistent. In a narrative review, Pincus and Morley (2001) 
ATTENTIONAL BIAS TO PAIN-RELATED INFORMATION

concluded that biases towards sensory pain stimuli, but not towards affective pain stimuli, are present in individuals with chronic pain. In an early meta-analysis of Stroop studies, Roelofs, Peters, Zeegers, and Vlaeyen (2002) found evidence of attentional biases towards both sensory and affective pain stimuli. Schoth, Nunes, and Liossi (2012) conducted a metaanalysis of dot probe studies specifically comparing individuals with chronic pain to healthy controls. These authors found evidence of attentional biases with both shorter $(300-500 \mathrm{~ms}$, Hedges $g=0.29)$ and longer $(1250 \mathrm{~ms}, g=0.42)$ stimulus presentation times. All of these reviews have been small, including only up to 10 attentional bias studies, prohibiting firm conclusions.

A more comprehensive meta-analysis has subsequently explored evidence for attentional biases to pain information across both pain and pain free populations (Crombez, Van Ryckeghem, Eccleston, \& Van Damme, 2013). When the studies that used the dot probe were investigated alone, it was concluded that attentional biases to pain related information existed in patients with chronic pain in comparison to healthy controls, but only for sensory pain word stimuli $(d=0.29)$. Therefore, it appears that attentional biases occur for those with chronic pain compared to those without, but that attentional biases are limited to sensory pain word stimuli when using the dot probe task. Although Crombez et al.'s (2013) meta-analysis is relatively recent, with the literature search being conducted in 2010, only a limited number of studies using the dot probe task were available at that time. Since then, the number of dot probe studies in this area has more than doubled, warranting the importance of an update of their meta-analysis. Further, Crombez et al.'s (2013) meta-analysis used data from several attentional bias tasks, and therefore could not address the effects of specific characteristics of the dot-probe task.

During the last decade, the dot-probe task has become the most widely used task to investigate attention bias, and overcomes some of the problems and limitations of earlier 
ATTENTIONAL BIAS TO PAIN-RELATED INFORMATION

tasks, such as the Stroop paradigm. Indeed, Stroop interference may arise from cognitive interference during worrying, or from a freezing response during the presentation of emotionally valenced stimuli (de Ruiter \& Brosschot, 1994; Karmann, Lautenbacher, \& Kunz, 2015; Nigg, 2000). The dot-probe therefore allows for more nuanced investigation of attentional bias parameters, compared with tasks such as the Stroop paradigm, which are likely to be at least partly accounting for general threat effects in addition to attentional bias (Algom, Chajut, \& Lev, 2004).

The overall objective of the present meta-analysis was to explore a) the factors driving attentional bias to pain (using the dot-probe task), and b) whether these biases are discriminative between different pain groups. Determining the conditions under which biases are present may help to understand their putative role in different populations, such as those anticipating pain and those experiencing acute and chronic pain. The dot-probe task has also been adapted for use as a therapeutic tool to modify attentional biases, which has shown promise for anxiety disorders (Jones \& Sharpe, 2017) and preliminary evidence for improving pain-related disability (Sharpe et al., 2012); however the mechanisms of change have been difficult to establish (Todd et al., 2015). A more detailed exploration of the dotprobe task may therefore assist in developing more accurate intervention tools. Further, determining whether attentional bias is consistently associated with the anticipation or experience of pain, may also help to identify individuals who are at risk of developing chronic pain. Indeed, a recent study found that individuals with greater attentional biases to affective pain words were more likely to go on to develop chronic pain (Sharpe, Haggman, Nicholas, Dear, \& Refshauge, 2014). The first aim was therefore to explore within which groups attentional biases are present. We conceptualised the pain experience according to the following groups: Currently experiencing pain (chronic pain, or acute pain), anticipating upcoming pain (anticipating either experimental or procedural pain), social concern for pain 
ATTENTIONAL BIAS TO PAIN-RELATED INFORMATION

(pain is present or anticipated in a close family member), or healthy (not currently in pain and not classified according to the other group categories). If attentional biases are related to the experience of pain, then we would expect that those currently experiencing pain would show greater attentional biases, at least to (sensory) pain stimuli, than healthy individuals. This view is supported by the meta-analysis of Crombez et al. (2013) and the model developed by Pincus and Morley (2001). If, on the other hand, attentional biases result from the salience of pain or the frequency with which people are exposed to pain-related stimuli, we would expect that those anticipating pain or with a social concern for pain would also exhibit attentional biases, and that these biases would be greater than in healthy individuals. Such a view could be supported by fear-avoidance models of pain, in which attentional hypervigilance may occur in anticipation of pain (Crombez et al., 2012; Vlaeyen \& Linton, 2000). If, however, attentional biases are a ubiquitous response to pain stimuli, then we would expect all groups, including healthy participants, to display attentional biases to pain stimuli. Theoretical models tend to argue that pain naturally captures attention (e.g. Eccleston \& Crombez, 1999), whereas attentional biases are often measured towards stimuli such as words and pictures. If we were to find attentional biases towards pain stimuli in all groups, it would extend current theoretical assumptions about attentional capture beyond actual pain. The second aim was to determine the parameters of the dot probe task that lead to expected differences in attentional biases between the pain groups where attentional biases are identified (e.g. patients with chronic pain), and healthy individuals. These parameters include those related to (a) the stimuli: type of (pain) stimulus, whether the stimuli are words or pictures, stimulus presentation duration, orientation of stimuli; (b) task procedure: task instructions given, intertrial intervals, proportion of pain trials, proportion of filler trials, whether gaze maintenance methods (such as eye-tracking or digit trials) were present, attentional target action required from participants, whether participants respond with one or two hands, and the tool used to 
record responses; and (c) the study itself: publication year, and overall study quality rating. Identifying such parameters can provide insights into the nature and underlying processes of attentional biases, such as whether the nature of attentional biases to sensory pain and affective pain information differ, how generalizable and robust attentional bias effects are, whether attentional biases are automatic processes, whether effects are more easily observed at early or late stages of attentional processing, and under which parameters we can best detect attentional bias effects. These findings will also help in designing a future generation of dot probe studies that can more reliably detect expected effects.

\section{Method}

\section{Literature search}

This meta-analysis was conducted following the PRISMA guidelines. Relevant published research was identified through two searches of the following electronic databases: MEDLINE, PsychINFO, and Web of Science. The first search was conducted on 14 October 2010 for an earlier meta-analysis (Crombez et al., 2013) with broad search terms: [selective attention* OR attention* bias* OR vigilance OR hypervigilance OR Stroop OR dot probe OR probe detection OR Posner OR spatial cueing OR spatial cuing] AND pain. From this original search, studies using the dot probe task and meeting the present inclusion and exclusion criteria were selected. The search was updated on 28 November 2017. As the present meta-analysis focused specifically on the dot-probe task (and not the Stroop or spatial cueing task), the search terms were narrowed to the following: [selective attention* OR attention* bias* OR vigilance OR hypervigilance OR dot probe OR probe detection] AND pain. In addition, key journals (e.g. PAIN, European Journal of Pain) and the references of relevant review articles were searched manually in order to determine if there were any missing studies. 
ATTENTIONAL BIAS TO PAIN-RELATED INFORMATION

\section{Inclusion and exclusion criteria}

Studies were included or excluded based on overall study factors, types of participants, and type of attentional bias task used. Only published studies were included in the present review, as (a) it may have been difficult to retrace and collect raw data of unpublished studies and (b) it was reasoned that studies that had not undergone peer review could not be guaranteed to be of a high quality. Where overlapping or identical data was reported in more than one manuscript, the data with the larger, more inclusive sample size was included, or, if they were identical, then the earliest publication of this data was used. Where necessary, study description information could be drawn from the related publications.

As we were only interested in attentional bias in adults, participants were required to be at least 18 years old. Where possible, if a study included some participants under 18 years of age, only individual participant data from those aged 18 years or older was included, as was the case for 11 studies (Boston \& Sharpe, 2005; Brookes, Sharpe, \& Dear, 2017; Dear, Sharpe, Nicholas, \& Refshauge, 2011b; Lautenbacher et al., 2010; McDermott, Peck, Walters, \& Smitherman, 2013; Sharpe et al., 2017; Sharpe, Johnson, \& Dear, 2015; Sharpe et al., 2010; Sharpe, Nicholson Perry, Rogers, Refshauge, \& Nicholas, 2013; Van Heck, Oosterman, de Kleijn, Jongsma, \& Van Rijn, 2017; Van Ryckeghem, Crombez, Van Hulle, \& Van Damme, 2012). The data and effect sizes for these studies may therefore differ from the published manuscripts. Where the raw data was not obtainable, studies were still included if the mean age of participants was 18 years or older (Bardel, Woodman, Perreaut-Pierre, \& Barizien, 2013; Lautenbacher et al., 2010).

Studies needed to include at least 20 participants per arm or pain group. For studies containing some groups with less than 20 participants and at least one group with 20 or more participants, the groups with 20 or more participants were still retained for the analyses 
ATTENTIONAL BIAS TO PAIN-RELATED INFORMATION

(Asmundson, Carleton, \& Ekong, 2005; Asmundson et al., 1997; Schoth \& Liossi, 2010). Further, if a study included a clinical intervention, only pre-intervention data was used (e.g. Bowler et al., 2017; Sharpe et al., 2012; Todd, Sharpe, \& Colagiuri, 2016). Finally, studies were required to investigate attentional biases in relation to pain-related information using the dot-probe task. The dot probe task consists of the presentation of a fixation point, followed by two visual stimuli that are presented simultaneously, either in the upper and lower, or left and right sections of the screen. One stimulus is of a category of interest (e.g. pain or threat), whilst the other is neutral or benign. The stimulus pair is immediately followed by a single visual target (or probe) in the same position as the stimulus of interest (congruent trials) or the same location as the neutral stimulus (i.e., opposite location to the stimulus of interest; incongruent trials). The task of the participants is to respond to the probe, or to one of its features (e.g. by a button press).

\section{Coding system}

The protocol was developed prior to conducting the present meta-analysis, detailing the aims, which key variables and moderators would be extracted and how they would be coded, and the quality rating criteria. The coding scheme was based on the coding system documented by Crombez et al. (2013), but was expanded to include specific methodological variables. These variables were determined by the authors, in collaboration with other members of the research laboratory group. An iterative process was used, whereby the authors read and provided feedback on the protocol until all authors were satisfied. The protocol was then pilot-tested on a selection of studies, and where the coding was ambiguous, the protocol was amended. All studies were then coded using this standardized procedure. The final protocol is available at http://hdl.handle.net/1854/LU-8560705. All coding was conducted independently by the first two authors, and any disagreements or ambiguity were 
ATTENTIONAL BIAS TO PAIN-RELATED INFORMATION

resolved through discussion to reach consensus. The final author was available for consultation during this process to ensure that the protocol was adhered to.

Pain categories were used to classify participants. The chronic pain group included participants who were categorised as such by the authors of the original study, with a minimum definition of pain occurring for at least 3 months. The acute pain group included participants who rated themselves as being in pain at present, but for less than 3 months. The anticipating pain group were participants who were healthy, but were either anticipating a subsequent painful task (e.g. cold pressor pain) as part of the research, or anticipating a painful medical procedure in the near future. The social concern for pain group included studies in which a close relative of the participant, such as a partner or child, experienced pain either as part of a pain condition or as an experimental task (e.g. cold pressor pain). Participants were classified as healthy if they did not meet criteria for any of the other groups, and were identified as part of a healthy control group by the authors of the study.

Stimuli were first categorised on stimulus type, based on whether the stimuli were words or pictures, and then on stimulus category. Stimuli were allocated to the following categories and subcategories (in parentheses) in preferential order: pain stimuli (sensory pain, affective pain, mixed pain); ill-health stimuli that were not specifically considered pain related by the authors of the included studies (illness, injury, disability, general health threat); threat stimuli that were not related to pain or ill-health (general threat, social threat, anger); positive stimuli. Furthermore, we coded the dot-probe task on stimulus presentation duration (subliminal, $<500 \mathrm{~ms}, 500-1000 \mathrm{~ms},>1000 \mathrm{~ms}$ ), and orientation of stimuli (up-down, leftright). Stimulus categorisation was conducted independently by two of the authors, and consensus was reached with no disagreements.

In addition, we coded information about the task procedure: (a) whether participants were given explicit instructions about the stimuli, such as using no explicit instructions, 
ATTENTIONAL BIAS TO PAIN-RELATED INFORMATION

participants told to attend to one stimulus (e.g. to read the top word of a pair; Asmundson et al., 2005), or participants told to attend to both stimuli (e.g. Dear, Sharpe, Nicholas, \& Refshauge, 2011a), (b) whether gaze maintenance methods (such as eye-tracking or digit trials used to assess focus on the task) were present, (c) whether the target action required probe detection (responding with one button based on whether or not a probe is present), probe localisation (responding with one of two buttons based on where the probe appears on the screen, e.g. on the left or right), or probe discrimination (responding with one of two buttons based on the type of probe, e.g. 'p' vs. 'q'), (d) response type (keyboard press, response box, other), (e) response method (one hand, two hands, no instructions).

Finally, we coded numerical information about the trials: inter-trial interval (ms), number of pain related trials, number of non-pain related trials, number of catch trials, number of gaze maintenance trials, and total number of trials, from which other measures such as proportion of pain trials and proportion of filler trials could be calculated. Additional information, such as publication year, was also extracted.

Studies were screened on internal and external quality rating criteria (see Supplementary File A) adapted from Crombez et al. (2013) and agreed upon by all authors. All studies were independently double coded on study quality, and then consensus was reached for all discrepancies through discussion. Publication bias for primary analyses was assessed with Egger's regression intercept and forest plots, and adjusted using Duval and Tweedie's trim and fill analysis. Finally, we conducted heterogeneity analyses to ensure the robustness of findings.

\section{Meta-analytic procedures}

Original datasets were obtained for $94 \%$ of the included studies. From these datasets, participants who were under 18 years of age were excluded. Attentional bias congruent and 
ATTENTIONAL BIAS TO PAIN-RELATED INFORMATION

incongruent reaction time means, the correlation between these means, standard deviations and sample size were calculated where possible from the original data. For the rest of the included studies, this information was obtained from the manuscripts. Correlations between congruent and incongruent reaction times were calculated across all included studies and an overall estimate of this correlation was derived $(r=0.899,95 \%$ CI $[0.884,0.911])$. This correlation was used as an estimate of association where actual correlations were not available.

Meta-analytic procedures were conducted using the Comprehensive Meta-Analysis program, version 3.3.070 (2014) . The primary effect of interest was the standardised paired difference (Cohen's $d$ for paired data) between congruent and incongruent mean reaction times, with a 95\% confidence interval. Cohen's (1992) guidelines were applied for small $(d=$ 0.1 to $d<0.5)$, medium $(d=0.5$ to $d<0.8)$, and large $(d \geq 0.8)$ effect sizes. In addition, an attentional bias index (i.e. the unstandardised paired difference between congruent and incongruent mean reaction times) was also calculated for main analyses, as this may be relevant for comparison for future researchers.

For all analyses, a random effects model was used to pool the data from different studies. A mixed effects model (i.e. a random-effects model within subgroups and a fixedeffect model across subgroups) was used to perform moderator analyses. Furthermore, due to a low number of studies in some subgroups it was opted to pool within group estimates of $\mathrm{Tau}^{2}$. Heterogeneity was assessed using the Q-value significance test, which provides an indicator of whether or not heterogeneity is present (Borenstein, Hedges, Higgins, \& Rothstein, 2009). Finally, for the continuous coded variables (e.g. inter-trial interval), we performed meta-regressions with a 2-tailed test using the methods of moments procedure and applied a Knapp-Hartung correction. 
ATTENTIONAL BIAS TO PAIN-RELATED INFORMATION

The analyses are structured in four parts. In the first part, we explored the overall attentional bias effects to pain words and pain pictures across all groups (chronic pain, acute pain, anticipating pain, or healthy). All pain word stimuli (e.g. sensory pain, affective pain, mixed pain) were grouped together to enable comparison with pain pictures, which are undifferentiated. In the second part, differences between the pain/non-pain groups were explored for each of the main word stimulus categories: sensory pain, affective pain, threat, ill-health, and positive. Some theoretical frameworks (e.g. Pincus \& Morley, 2001), suggest a distinction between sensory and affective pain stimuli, and therefore these stimuli were analysed separately for subsequent analyses. In the third part, moderator analyses were conducted for pain stimuli in groups where heterogeneity was identified, and where there were at least three studies within each parameter group, to reduce the risk of bias. In the fourth part, the chronic pain and healthy groups were compared for sensory pain words, within each of the parameter categories where there were at least three studies present. Sensory pain words were focused upon because this was the pain stimulus that was found to have the strongest effects in the previous meta-analysis (Crombez et al., 2013).

\section{Results}

\section{Study selection}

The initial search retrieved 1,138 references, with the update retrieving an additional 1030 references. Titles, abstracts, and full text articles from the available literature were screened for inclusion in the review. Records were independently screened by two raters, and as no studies were found to have been missed in the screening process, the screening was considered satisfactory.

Studies were screened out based on the exclusion criteria described, as detailed in Supplementary File B. After exclusion, there was a final selection of 52 articles $(\mathrm{N}=4466)$. 
ATTENTIONAL BIAS TO PAIN-RELATED INFORMATION

Thirty-four $(65 \%)$ of these articles $(N=2887)$ were new since the meta-analysis of Crombez et al. (2013) confirming the need for a novel meta-analysis investigating attentional bias for pain related information. The search flowchart is provided in Figure 1, and a list of the included studies can be found in Supplementary File C.

\section{INSERT FIGURE 1 NEAR HERE}

\section{Descriptive statistics}

There were three studies reporting on participants experiencing acute pain $(n=204)$, 17 studies reporting on patients with chronic pain $(n=1099)$, nineteen studies reporting on people anticipating pain (anticipating experimental pain $k=16, n=1349$; anticipating procedural pain $k=3, n=160)$, and 27 studies reporting on a healthy sample $(n=1603)$. As only one study $(n=52)$ fell within the social concern for pain category (Vervoort et al., 2011), this study was excluded from further analyses. For further information about the study characteristics, see Supplementary File D.

\section{Quality analysis}

On average, studies fulfilled $72 \%$ of the external validity criteria and $49 \%$ of the internal validity criteria. The low internal validity was primarily due to inadequate methods for accounting for task engagement (19.6\% of studies), and not sufficiently accounting for the relevance of the pain-related stimuli to the sample (39.2\% of studies). Detailed information on the quality ratings can be found in Supplementary File A. 
ATTENTIONAL BIAS TO PAIN-RELATED INFORMATION

\section{Pain word and picture stimuli}

Attentional biases to pain word and pain picture stimuli were explored across the pain groups, as displayed in Table 1. For pain words, there was a significant effect overall $(d=$ $0.060, p=.002)$. This effect did not change when Duval and Tweedie's trim and fill method was used to adjust the effect to account for potential missing studies to the left of the mean ( $d$ $=0.060,95 \% \mathrm{CI}[0.020,0.100])$. There was a significant effect for chronic pain patients $(d=$ $0.136, p=.002)$. With Duval and Tweedie's trim and fill analysis to adjust for missing studies to the left of the mean, the effect remained similar $(d=0.138,95 \%$ CI $[0.033$, 0.243]). There was no significant effect of attentional bias to pain words for acute pain patients $(d=0.127, p=.119)$, those anticipating pain $(d=0.041, p=.173)$, or healthy individuals $(d=0.022, p=.541)$. For pain pictures, the overall effect was not significant $(d=$ $0.029, p=.339)$, and remained consistent when Duval and Tweedie's trim and fill was used to the left of the mean $(d=0.029,95 \%$ CI $[-0.030,0.087])$. There was a significant effect for chronic pain patients $(d=0.110, p=.045)$, and again using Duval and Tweedie's trim and fill to the left of the mean, the effect remained similar $(d=0.110,95 \%$ CI $[0.002,0.217])$. There was no significant effect of attentional bias towards pain pictures for those anticipating pain $(d=0.054, p=.347)$ or healthy individuals $(d=-0.044, p=.337)$. There were no studies using pain pictures within an acute pain sample.

INSERT TABLE 1 NEAR HERE 


\section{Comparing pain groups for each type of word stimuli}

The second set of analyses compared pain groups (chronic pain, acute pain, anticipating pain, and healthy) for each type of word stimuli (sensory pain, affective pain, threat, ill-health, and positive), as displayed in Table 2.

\section{INSERT TABLE 2 NEAR HERE}

The overall within-groups effect for sensory pain words across all groups was small but significant $(d=.083, p=.001)$, also reflected in the small mean attentional bias index $(M$ $=3.103 \mathrm{~ms} ; S E=0.88$ ). This effect did not change when Duval and Tweedie's trim and fill analysis was used to estimate missing studies to the left of the mean $(d=0.083,95 \% \mathrm{CI}$ $[0.028,0.137])$. Follow-up analyses indicated that effect-sizes differed significantly between groups $\left(Q_{3}=12.61, p=.006\right)$. There was a significant attentional bias toward sensory pain information for those with chronic pain $(d=0.198, p<.001)$, with an average attentional bias index of $9.143 \mathrm{~ms}(S E=2.07)$. Using Duval and Tweedie's trim and fill to the left, this effect remained similar $(d=0.196,95 \%$ CI $[0.056,0.336])$. There was significant heterogeneity $\left(Q_{11}=36.28, p<.001\right)$ between effect sizes, warranting follow-up moderator analyses. The effect for people experiencing acute pain was also significant $(d=0.303, p=$ $.003)$, with an average attentional bias index of $13.901 \mathrm{~ms}(S E=3.76)$. Again, significant heterogeneity was found between the effect sizes $\left(Q_{2}=12.60, p=.002\right)$. The effect sizes and heterogeneity between effect sizes of studies including people anticipating pain $(d=0.038, p$ $\left.=.339 ; Q_{17}=16.22, p=.508\right)$, and healthy participants $\left(d=0.012, p=.841 ; Q_{16}=24.97, p\right.$ $=.070)$ were not significant. See Supplementary File E for more information on bias statistics for primary analyses, including forest plots and Egger's regression test. 
ATTENTIONAL BIAS TO PAIN-RELATED INFORMATION

The overall effect for threat words was not significant $(d=-0.012, p=.746)$, and neither was the difference between groups $\left(Q_{3}=6.09, p=.107\right)$. Results did however show significant heterogeneity between effect sizes for samples of patients with chronic pain $\left(Q_{7}=\right.$ $30.71, p<.001)$, samples of patients with acute pain $\left(Q_{2}=8.55, p=.014\right)$, and samples of healthy individuals $\left(Q_{11}=23.66, p=.014\right)$. For affective pain words, ill-health words, and positive words, no significant overall or between groups effect-sizes were found, and there was no significant heterogeneity for any of the subgroups.

As the focus of this meta-analysis is on attentional biases for pain-related information, we opted to further investigate heterogeneity in effect sizes of studies investigating attentional bias towards sensory pain words within the chronic pain group. There were insufficient studies $(k=3)$ to conduct further analyses within the acute pain group.

\section{Chronic pain group: Moderators of attentional bias towards sensory pain words}

We planned to investigate the following categorical moderators: stimulus orientation, stimulus presentation time, task instructions about stimulus processing, presence of gaze maintenance methods, target action required upon probe presentation, type of response tool/equipment, and method of response (i.e., whether participants used one or two hands to respond). However, as researchers have often used similar task parameters across studies using sensory pain words, there were insufficient studies $(k<3)$ in the comparison groups to conduct the planned analyses. The exceptions were target action and stimulus presentation time. For target action, there was a significant effect for studies using probe discrimination $(d$ $=0.239, p=.023)$, but not for studies using probe localisation $(d=0.168, p=.239)$.

However, the difference was not significant $\left(Q_{1}=0.16, p=.691\right)$. For stimulus presentation times, there was a significant effect for studies presenting the word stimuli for 500-1000 ms 
ATTENTIONAL BIAS TO PAIN-RELATED INFORMATION

$(d=0.205, p=.029)$, but not for studies presenting the word stimuli for $>1000 \mathrm{~ms}(d=$ $0.256, p=.141)$. Again, the difference was not significant $\left(Q_{1}=0.07, p=.797\right)$.

In addition, five continuous moderators were investigated within a single metaregression: article publication year, proportion of pain trials, proportion of non-active (neutral-neutral, digit, catch) trials, inter-trial interval, and the quality rating. The overall meta-regression model was not significant $\left(F_{5,11}=1.17, p=.420\right)$, and none of the variables independently accounted for variance in attentional bias. Although there was a moderately high variance inflation factor for proportion of pain trials, potentially suggesting multicollinearity and overlap with proportion of non-active trials, running the analyses separately for each variable produced an identical pattern of results. See Supplementary File F for further information.

\section{Comparing chronic pain and healthy groups across parameters for sensory pain words}

Previous analyses clearly showed that chronic pain patients only show an attention bias towards sensory pain words. To further address under which circumstances (i.e., with which parameters) attentional bias for sensory pain words differs between chronic pain patients and healthy volunteers, we calculated the effect sizes for each parameter separately for those in the chronic pain group and the healthy group, and where possible (i.e. when there were at least three studies in both groups), compared the effect sizes. The results, including confidence intervals, are presented in Table 3. Effect sizes were able to be compared for the following parameters: upper-lower stimulus orientation, 500-1000ms stimulus presentation time, $>1000 \mathrm{~ms}$ stimulus presentation time, task instructions to process both stimuli, gaze maintenance absent, target action as probe discrimination, target action as probe localisation, keyboard as the response tool, and method of response with two hands. Where it was not possible to compare effect sizes due to a small number of studies, the effects were still 
ATTENTIONAL BIAS TO PAIN-RELATED INFORMATION

included in Table 3 to provide information for future researchers who may wish to use some of these parameters, despite the lack of firm effects yet.

\section{INSERT TABLE 3 NEAR HERE}

Results indicated that when using an upper-lower stimulus orientation, there was a significantly larger attentional bias towards sensory pain words for the chronic pain group ( $d$ $=0.237, p=.002)$ compared to the healthy group $\left(d=-0.015, p=.713 ; Q_{1}=8.462, p=\right.$ $.004)$. For stimulus presentation time of $500-1000 \mathrm{~ms}$, there was also a significantly larger effect for the chronic pain group $(d=0.212, p=.003)$ compared to the healthy group $(d=$ $\left.0.031, p=.455 ; Q_{1}=4.901, p=.027\right)$. However, for presentations greater than $1000 \mathrm{~ms}$, there was no significant difference between the chronic pain group $(d=0.270, p=.387)$ and the healthy group $\left(d=0.045, p=.715 ; Q_{1}=0.448, p=.503\right)$. When participants were instructed to attend to both stimuli, the effect sizes were not significantly different between the chronic pain group $(d=0.219, p=0.010)$ and the healthy group $\left(d=0.084, p=0.08 ; Q_{1}\right.$

$=1.806, p=.179)$. When gaze maintenance was absent, there was a significantly greater attentional biases for the chronic pain group $(d=0.237, p=.002)$ compared to the healthy group $\left(d=-0.007, p=.866 ; Q_{1}=8.168, p=.004\right)$. When target action involved probe discrimination, there was no significant difference between those in the chronic pain group ( $d$ $=0.239, p=.019)$ and the healthy group $\left(d=.053, p=.491 ; Q_{1}=2.103, p=.147\right)$; and for

probe localisation there was also no significant difference between the chronic pain group $(d$ $=0.170, p=.275)$ and the healthy group $\left(d=0.022, p=.687 ; Q_{1}=0.811, p=.368\right)$. When a keyboard was the response tool, there was no significant difference between attentional bias effects in the chronic pain group $(d=0.167, p=.020)$ and the healthy group $(d=-0.011, p=$ $\left..849 ; Q_{1}=3.795, p=.051\right)$. Finally, when participants used two hands to respond to the probe 
ATTENTIONAL BIAS TO PAIN-RELATED INFORMATION

there was no significant difference between the chronic pain group $(d=0.214, p=.009)$ and the healthy group $\left(d=0.045, p=.347 ; Q_{1}=3.214, p=.073\right)$.

\section{Discussion}

The present meta-analysis investigated the existence of attentional biases (assessed using the dot-probe task) in different (pain) populations, and explored the impact of pivotal theoretical and methodological variables. Overall, patients with chronic pain and acute pain displayed a small but significant attentional bias towards sensory pain words that was maintained across many parameters of the dot probe task. These effects were not found for those anticipating pain or healthy individuals; nor were they found for other types of stimuli, such as affective pain, threat, ill-health, or positive words. The parameters for which biases to sensory pain words were found with chronic pain patients included: instructing participants to process both stimuli, using an upper-lower stimulus orientation, presenting the stimuli for 500-1000ms, not using gaze maintenance methods, using probe discrimination, and responding with either a keyboard press or response box using two hands.

Our pattern of results is in keeping with previous reviews by Crombez et al. (2013) and Pincus and Morley (2001). The overall effect for sensory pain biases within chronic pain patients using the dot probe task $(d=0.196)$ was slightly smaller than the effect $(d=0.292)$ found by Crombez et al. (2013), however this effect remained significantly larger than the effect observed in healthy individuals, indicating that this effect is relatively robust. These findings together suggest that the sensory representations of pain are important when it comes to capturing attention in patients with chronic pain, rather than affective representations (as would be detected by affective pain stimuli, or even potentially threat or positive word stimuli). Interestingly, researchers have posited that an attentional bias towards positive words or away from affective pain words is a risk factor for chronic pain, in that attentional 
ATTENTIONAL BIAS TO PAIN-RELATED INFORMATION

bias towards positive words may indicate poor coping and dysfunctional pain avoidance (Lautenbacher et al., 2010; Todd et al., 2015). Current findings provide no support for this view.

The most parsimonious explanation for the presence of biases in those with acute and chronic pain (and not in those free of pain) is that attentional bias is a feature or consequence of the pain experience. This view is supported by theoretical models such as the schema enmeshment model (Pincus \& Morley, 2001), whereby individuals develop enmeshed pain schemas leading to cognitive biases as a result of an ongoing pain experience. This is also consistent with the misdirected problem solving model (Eccleston \& Crombez, 2007), where those with pain are thought to prioritise pain, becoming 'stuck' in an effort to solve the problem of pain, at the expense of other life goals. Accordingly, attentional bias may then best viewed as a process involved in the maintenance or exacerbation of pain problems. In line with this view, attentional bias has been found to predict and explain later pain outcomes (see Todd et al., 2015), although relatively few studies have addressed this idea, and further research is needed to corroborate these findings.

That only those experiencing pain display an attentional bias towards pain information may seem at odds with the notion that attentional bias is a normal phenomenon that will emerge in everyone who anticipates pain (Van Damme, Legrain, Vogt, \& Crombez, 2010). This finding is also in contrast with the idea that individuals often display attentional biases to stimuli that are immediately relevant for the pursuit of their goals (Vogt, De Houwer, Moors, Van Damme, \& Crombez, 2010), such as the anticipation of or attempt to control actual pain (Crombez et al., 2013; Notebaert et al., 2011). It may well be that the unique presence of an attention bias for pain-related information in people experiencing pain, but not in those anticipating pain is specific for tasks that use symbolic stimuli. In healthy 
individuals, linguistic representations of pain may be insufficient to generate a bias in the absence of a goal to reduce or avoid pain.

Doubts have been raised about whether symbolic stimuli, such as pain words, are able to automatically activate the relevant pain memory or schema, an assumption underlying the phenomenon of attentional bias (Van Ryckeghem \& Crombez, 2018). The observation that more than half of the studies in our meta-analysis did not sufficiently account for the relevance of the pain-related stimuli to the sample may add to this problem. Indeed, it is reasonable to assume that only words unambiguously related to the experience of pain may elicit an attentional bias. Some words may be generally accepted as pain relevant (e.g. the word 'pain'). Other words may be specific to a pain syndrome, or even to an individual's experience (Andersson \& Haldrup, 2003; Crombez, Hermans, \& Adriaensen, 2000; Dear et al., 2011a). This problem may have reduced the magnitude of the effect size of attentional bias towards pain-related information in current meta-analysis. When researchers wish to pursue words or pictures as pain-related information we strongly recommend documenting how strongly their stimuli are associated with pain. Existing techniques may be easily adapted for this purpose (Fazio, Williams, \& Powell, 2000), but have seldom been used in the area of cognitive biases (see Ferguson, Moghaddam, and Bibby (2007) for an exception). Another avenue of research is to develop somatosensory versions of attentional bias tasks (Crombez, Heathcote, \& Fox, 2015; Van Ryckeghem \& Crombez, 2014; Van Ryckeghem \& Crombez, 2018; Van Ryckeghem \& Vervoort, 2016). One such variant of an attentional bias task was included in the meta-analysis of Crombez et al. (2013), i.e. a modified spatial cueing paradigm in which the cues were made predictive of actual pain by means of a classical conditioning procedure (e.g. Van Damme et al., 2004; Van Ryckeghem et al., 2012). However, researchers have recently been exploring and developing alternatives (Van Damme, Bulcke, Van Den Berghe, Poppe, \& Crombez, 2018). Important challenges will be 
ATTENTIONAL BIAS TO PAIN-RELATED INFORMATION

to make these tasks relevant for the pain of patients, and to achieve an acceptable level of reliability in order to investigate the role of individual difference variables.

To our surprise, we were unable to perform many of the planned moderator analyses. This was largely due to the lack of variation in task parameters of the dot probe task. Over the years, researchers seem to have favoured a particular set of parameters to study attentional bias in pain. From the studies included, these common parameters included using word stimuli, not giving explicit instructions about stimulus processing or instructing participants to process both stimuli, upper-lower stimulus orientation, presenting the stimuli for 500$1000 \mathrm{~ms}$, not including gaze maintenance methods, using probe localisation or more recently using probe discrimination, and responding with a keyboard press using two hands. Many of these parameters were found to result in significant effects for chronic pain patients in comparison to healthy individuals in the present research: upper-lower stimulus orientation, presenting the stimuli for 500-1000ms, and not including gaze maintenance methods; whilst others may not have resulted in significant differences due to smaller numbers of studies and therefore less power. These parameters may indicate under which conditions pain-relevant attentional biases can be observed. However, with many studies using the same parameters, it is difficult to determine which particular parameters may influence the effects, and which are redundant. Future research could aim to disentangle the impact of these commonly combined parameters by systematically varying them.

It is generally assumed that the tasks used to measure attentional bias (such as the dotprobe task) are valid, in that they actually measure "attentional bias". However, changing task parameters in order to optimise the effect size may have unintended effects, as doing so may result in changes to the underlying process being assessed. For example, the process being measured at subliminal stimulus presentation lengths (vigilance) may be different to the process being measured when stimuli are presented for 1000ms (difficulty disengaging). 
ATTENTIONAL BIAS TO PAIN-RELATED INFORMATION

Also, in many studies researchers have begun to instruct participants to read both word stimuli, hence making attention to these stimuli task relevant. It is likely that in such situations intentional and strategic processes may come more to the fore.

The overall quality of the studies has not improved since the meta-analysis of Crombez et al. (2013), with current ratings of $72 \%$ and $49 \%$ for external and internal validity, respectively, compared with $70 \%$ and $57 \%$ in the previous meta-analysis. One of the most notable limitations of the selected studies was the lack of information about the pain experience, particularly in the control group. Current pain level was often not reported, but is essential even within healthy samples given that pain is highly prevalent, and a majority of people will experience an episode of pain within a 6 month period (Hastie, Riley, \& Fillingim, 2005). In addition, most studies did not adequately assess relevance of the painrelated information to that sample. As such, the content validity of the stimulus material used is currently poor. Finally, very few accounted for task engagement, which can help to increase the validity of the task. One way is to use eye-tracking methodology, which can both ensure eye gaze focus on the task, as well as providing a more direct measure of task engagement through eye tracking data (e.g. Sharpe et al., 2017; Yang, Jackson, \& Chen, 2013; Yang, Jackson, Gao, \& Chen, 2012).

Researchers have begun to directly target attentional bias in clinical interventions. There is good evidence for the effectiveness of attentional bias modification procedures for improving a range of conditions (MacLeod, 2012), particularly for anxiety disorders (BarHaim, 2010; Jones \& Sharpe, 2017). However, this area is not without controversy, as the tasks purported to modify attentional bias do not do so consistently (Koster \& Bernstein, 2015). As yet, systematic research on attentional bias modification in chronic pain is (just) emerging. There are some initial and promising results, although mechanisms of change have been harder to identify (Sharpe et al., 2012; Todd et al., 2015). More carefully designed, 
ATTENTIONAL BIAS TO PAIN-RELATED INFORMATION

registered trials are needed in this area. Further, we need more research to determine whether attentional bias is an authentic causal factor in the development or exacerbation of pain problems, as with anxiety disorders (Van Bockstaele et al., 2014). This is not merely an intellectual and theoretical endeavour. If attentional bias is proved to be driven by the appraisal of pain as a threatening experience, other therapeutic techniques may work equally well, including cognitive-behavioural techniques (Dehghani, Sharpe, \& Nicholas, 2004; Van Ryckeghem \& Crombez, 2014).

There are some limitations to this meta-analysis. As only cross-sectional research was included, the direction of causality between attentional bias and the chronic pain experience is unclear. Further, many of the parameters investigated were interrelated, and multicollinearity occurred in some instances. It is therefore not always clear which specific parameters accounted for the effects observed or whether their combination is important. In addition, some groups (such as those with acute pain and those with social concern for pain) and parameters remain underrepresented, meaning that the planned moderators could not be adequately assessed. Therefore, some of our conclusions are preliminary and await further corroboration. It is also worth noting that the classification of stimuli into categories such as sensory pain, health, and threat was retained from the original studies, and as such may not always represent the most accurate or conceptually sound categorisation of stimuli. We strongly recommend that researchers pilot-test stimuli in a relevant sample to ensure that they fit the category of interest prior to using them in the dot-probe task and other attentional bias measures. Finally, we were unable to look at the role of individual differences in attentional bias. It may well be that some effects are only found in groups of individuals with particular characteristics such as pain catastrophizing, pain-related fear, or threatening pain interpretations; although some of these individual differences were investigated by Crombez et al. (2013) with no effects. 


\section{Conclusions}

This meta-analysis containing 52 articles found small but consistent evidence for attentional biases towards pain words and pain pictures for individuals with chronic pain, evidence for attentional bias towards sensory pain words for individuals with chronic and acute pain, and also evidence for the discriminative nature of attentional bias in differentiating between individuals with chronic pain and those without, when sensory pain words were used. We make important recommendations for future research. Firstly, it is recommended that sensory pain stimuli is used to investigate the nature of attentional biases that are observed with patients with chronic pain, and that combining sensory pain stimuli with other kinds of stimuli such as affective pain, threat, and ill-health stimuli into a single category is likely to weaken the effects. Secondly, biases to symbolic representations of pain (i.e. words) are likely to be observed in patients with chronic and acute pain, but not healthy individuals, regardless of whether or not they are anticipating pain. Taken together, these findings point towards the role of sensory aspects of the pain experience, rather than the emotional content, in producing pain-related attentional biases. Finally, researchers should try to explicitly vary different parameters (in line with the theoretical understanding of attentional biases). In addition, more research with those with acute pain and a social concern for pain is needed to determine the effect on attentional biases so that we can improve the evidence base further in this area. 
ATTENTIONAL BIAS TO PAIN-RELATED INFORMATION

\section{References}

Aldrich, S., Eccleston, C., \& Crombez, G. (2000). Worrying about chronic pain: Vigilance to threat and misdirected problem solving. Behaviour Research and Therapy, 38(5), 457-470. doi:10.1016/S0005-7967(99)00062-5

Algom, D., Chajut, E., \& Lev, S. (2004). A rational look at the emotional stroop phenomenon: A generic slowdown, not a stroop effect. Journal of Experimental Psychology: General, 133(3), 323-338. doi:10.1037/0096-3445.133.3.323

Andersson, G., \& Haldrup, D. (2003). Personalized pain words and Stroop interference in chronic pain patients. European Journal of Pain, 7(5), 431-438. doi:10.1016/S10903801(03)00002-8

Asmundson, G. J., Carleton, R. N., \& Ekong, J. (2005). Dot-probe evaluation of selective attentional processing of pain cues in patients with chronic headaches. Pain, 114(1), 250-256. doi:10.1016/j.pain.2004.12.025

Asmundson, G. J. G., Kuperos, J. L., \& Ron Norton, G. (1997). Do patients with chronic pain selectively attend to pain-related information?: Preliminary evidence for the mediating role of fear. Pain, 72(1-2), 27-32. doi:10.1016/S0304-3959(97)00010-9

Bar-Haim, Y. (2010). Research Review: Attention bias modification (ABM): A novel treatment for anxiety disorders. Journal of Child Psychology and Psychiatry, 51(8), 859-870. doi:10.1111/j.1469-7610.2010.02251.x

Bar-Haim, Y., Lamy, D., Pergamin, L., Bakermans-Kranenburg, M. J., \& van Ijzendoorn, M. H. (2007). Threat-related attentional bias in anxious and nonanxious individuals: A meta-analytic study. Psychological Bulletin, 133(1), 1-24. doi:10.1037/00332909.133.1.1 
ATTENTIONAL BIAS TO PAIN-RELATED INFORMATION

Bardel, M. H., Woodman, T., Perreaut-Pierre, E., \& Barizien, N. (2013). The role of athletes' pain-related anxiety in pain-related attentional processes. Anxiety, Stress, \& Coping, 26(5), 573-583. doi:10.108010615806.2012.757306

Borenstein, M., Hedges, L. V., Higgins, J. P. T., \& Rothstein, H. R. (2009). Introduction to meta-analysis. West Sussex, UK: Wiley.

Boston, A., \& Sharpe, L. (2005). The role of threat-expectancy in acute pain: Effects on attentional bias, coping strategy effectiveness and response to pain. Pain, 119(1-3), 168-175. doi:10.1016/j.pain.2005.09.032

Bowler, J., Bartholomew, K., Kellar, I., Mackintosh, B., Hoppitt, L., \& Bayliss, A. (2017). Attentional bias modification for acute experimental pain: A randomized controlled trial of retraining early versus later attention on pain severity, threshold and tolerance. European Journal of Pain, 21(1), 112-124. doi:10.1002/ejp.908

Brookes, M., Sharpe, L., \& Dear, B. (2017). Rumination induces a pattern of attention characterized by increased vigilance followed by avoidance of affective pain words. European Journal of Pain, 21(7), 1197-1208. doi:10.1002/ejp.1020

Brooks, S., Prince, A., Stahl, D., Campbell, I. C., \& Treasure, J. (2011). A systematic review and meta-analysis of cognitive bias to food stimuli in people with disordered eating behaviour. Clinical Psychology Review, 31(1), 37-51. doi:10.1016/j.cpr.2010.09.006

Bundesen, C. (1990). A theory of visual attention. Psychological Review, 97(4), 523. doi:10.1037/0033-295X.97.4.523

Cohen, J. (1992). A power primer. Psychological Bulletin, 112(1), 155-159. doi:10.1037/0033-2909.112.1.155

. Comprehensive Meta Analysis (Version 3.3.070). (2014). Inglewood, NJ, USA: Biostat Inc. Retrieved from http://www.comprehensive.com 
ATTENTIONAL BIAS TO PAIN-RELATED INFORMATION

Crombez, G., Eccleston, C., Van Damme, S., Vlaeyen, J. W., \& Karoly, P. (2012). Fearavoidance model of chronic pain: The next generation. The Clinical Journal of Pain, 28(6), 475-483. doi:10.1097/AJP.0b013e3182385392

Crombez, G., Heathcote, L., \& Fox, E. (2015). The puzzle of attentional biases to pain: Beyond attention. Pain, 156(9), 1581-1582. doi:10.1097/j.pain.0000000000000255

Crombez, G., Hermans, D., \& Adriaensen, H. (2000). The emotional stroop task and chronic pain: What is threatening for chronic pain sufferers? European Journal of Pain, 4(1), 37-44. doi:10.1053/eujp.1999.0149

Crombez, G., Van Ryckeghem, D., Eccleston, C., \& Van Damme, S. (2013). Attentional bias to pain-related information: A meta-analysis. Pain, 154(4), 497-510. doi:10.1016/j.pain.2012.11.013

de Ruiter, C., \& Brosschot, J. F. (1994). The emotional stroop interference effect in anxiety: attentional bias or cognitive avoidance? Behaviour Research and Therapy, 32(3), 315319. doi:10.1016/0005-7967(94)90128-7

Dear, B. F., Sharpe, L., Nicholas, M. K., \& Refshauge, K. (2011a). Pain-related attentional biases: The importance of the personal relevance and ecological validity of stimuli. The Journal of Pain, 12(6), 625-632. doi:10.1016/j.jpain.2010.11.010

Dear, B. F., Sharpe, L., Nicholas, M. K., \& Refshauge, K. (2011b). The psychometric properties of the dot-probe paradigm when used in pain-related attentional bias research. The Journal of Pain, 12(12), 1247-1254. doi:10.1016/j.jpain.2011.07.003

Dehghani, M., Sharpe, L., \& Nicholas, M. (2004). Modification of attentional biases in chronic pain patients: A preliminary study. European Journal of Pain, 8(6), 585-594. doi:10.1016/j.ejpain.2004.02.003

Donders, F. C. (1969). On the speed of mental processes. Acta Psychologica, 30, 412-431. doi:10.1016/0001-6918(69)90065-1 
ATTENTIONAL BIAS TO PAIN-RELATED INFORMATION

Eccleston, C., \& Crombez, G. (1999). Pain demands attention: A cognitive-affective model of the interruptive function of pain. Psychological Bulletin, 125(3), 356-366. doi: $10.1037 / 0033-2909.125 .3 .356$

Eccleston, C., \& Crombez, G. (2007). Worry and chronic pain: A misdirected problem solving model. Pain, 132(3), 233-236. doi:10.1016/j.pain.2007.09.014

Fazio, R. H., Williams, C. J., \& Powell, M. C. (2000). Measuring associative strength: Category $\square$ item associations and their activation from memory. Political Psychology, 21(1), 7-25. doi:10.1111/0162-895X.00175

Ferguson, E., Moghaddam, N. G., \& Bibby, P. A. (2007). Memory bias in health anxiety is related to the emotional valence of health-related words. Journal of Psychosomatic Research, 62(3), 263-274. doi:10.1016/j.jpsychores.2007.01.015

Field, M., Munafò, M. R., \& Franken, I. H. (2009). A meta-analytic investigation of the relationship between attentional bias and subjective craving in substance abuse. Psychological Bulletin, 135(4), 589-607. doi:10.1037/a0015843

Gatchel, R. J., Peng, Y. B., Peters, M. L., Fuchs, P. N., \& Turk, D. C. (2007). The biopsychosocial approach to chronic pain: scientific advances and future directions. Psychological Bulletin, 133(4), 581-624. doi:10.1037/0033-2909.133.4.581

Gotlib, I. H., Krasnoperova, E., Yue, D. N., \& Joormann, J. (2004). Attentional biases for negative interpersonal stimuli in clinical depression. Journal of Abnormal Psychology, 113(1), 127-135. doi:10.1037/0021-843X.113.1.127

Hastie, B. A., Riley, J. L., \& Fillingim, R. B. (2005). Ethnic differences and responses to pain in healthy young adults. Pain Medicine, 6(1), 61-71. doi:10.1111/j.15264637.2005.05009.x

Hendrikse, J., Cachia, R., Kothe, E., McPhie, S., Skouteris, H., \& Hayden, M. (2015). Attentional biases for food cues in overweight and individuals with obesity: A 
ATTENTIONAL BIAS TO PAIN-RELATED INFORMATION

systematic review of the literature. Obesity Reviews, 16(5), 424-432. doi:10.1111/obr.12265

Hou, R., Moss-Morris, R., Bradley, B. P., Peveler, R., \& Mogg, K. (2008). Attentional bias towards health-threat information in chronic fatigue syndrome. Journal of Psychosomatic Research, 65(1), 47-50. doi:10.1016/j.brat.2013.10.005

Johannes, C. B., Le, T. K., Zhou, X., Johnston, J. A., \& Dworkin, R. H. (2010). The prevalence of chronic pain in United States adults: results of an Internet-based survey. The Journal of Pain, 11(11), 1230-1239. doi:10.1016/j.jpain.2010.07.002

Jones, E. B., \& Sharpe, L. (2017). Cognitive Bias Modification: A review of meta-analyses. Journal of Affective Disorders, 223, 175-183. doi:10.1016/j.jad.2017.07.034

Karmann, A. J., Lautenbacher, S., \& Kunz, M. (2015). The role of inhibitory mechanisms in the regulation of facial expressiveness during pain. Biological Psychology, 104, 8289. doi:10.1016/j.biopsycho.2014.11.016

Koster, E. H. W., \& Bernstein, A. (2015). Introduction to the special issue on Cognitive bias modification: Taking a step back to move forward? Journal of Behavior Therapy and Experimental Psychiatry, 49, Part A, 1-4. doi:10.1016/j.jbtep.2015.05.006

Lautenbacher, S., Huber, C., Schöfer, D., Kunz, M., Parthum, A., Weber, P. G., Roman, C., Griessinger, N., \& Sittl, R. (2010). Attentional and emotional mechanisms related to pain as predictors of chronic postoperative pain: A comparison with other psychological and physiological predictors. Pain, 151(3), 722-731.

doi:10.1016/j.pain.2010.08.041

Leadley, R., Armstrong, N., Lee, Y., Allen, A., \& Kleijnen, J. (2012). Chronic diseases in the European Union: the prevalence and health cost implications of chronic pain. Journal of Pain \& Palliative Care Pharmacotherapy, 26(4), 310-325.

doi:10.3109/15360288.2012.736933 
ATTENTIONAL BIAS TO PAIN-RELATED INFORMATION

MacLeod, C. (2012). Cognitive bias modification procedures in the management of mental disorders. Current Opinion in Psychiatry, 25(2), 114-120. doi:10.1097/YCO.0b013e32834fda4a

MacLeod, C., Mathews, A., \& Tata, P. (1986). Attentional bias in emotional disorders. Journal of Abnormal Psychology, 95(1), 15-20. doi:10.1037/0021-843X.95.1.15

Macmahon, K. M., Broomfield, N. M., \& Espie, C. A. (2006). Attention bias for sleep-related stimuli in primary insomnia and delayed sleep phase syndrome using the dot-probe task. Sleep, 29(11), 1420-1427. doi:10.1093/sleep/29.11.1420

Mathews, A., \& MacLeod, C. (1985). Selective processing of threat cues in anxiety states. Behaviour Research and Therapy, 23(5), 563-569. doi:10.1016/0005-7967(85)901044

McDermott, M. J., Peck, K. R., Walters, A., \& Smitherman, T. A. (2013). Do episodic migraineurs selectively attend to headache-related visual stimuli? Headache: The Journal of Head and Face Pain, 53(2), 356-364. doi:10.1111/head.12011

Nigg, J. T. (2000). On inhibition/disinhibition in developmental psychopathology: views from cognitive and personality psychology and a working inhibition taxonomy. Psychological Bulletin, 126(2), 220. doi:10.1037//0033-2909.126.2.220

Notebaert, L., Crombez, G., Vogt, J., De Houwer, J., Van Damme, S., \& Theeuwes, J. (2011). Attempts to control pain prioritize attention towards signals of pain: an experimental study. Pain, 152(5), 1068-1073. doi:10.1016/j.pain.2011.01.020

Pearce, J., \& Morley, S. (1989). An experimental investigation of the construct validity of the McGill Pain Questionnaire. Pain, 39(1), 115-121. doi:10.1016/0304-3959(89)90182-6

Pincus, T., \& Morley, S. (2001). Cognitive-processing bias in chronic pain: A review and integration. Psychological Bulletin, 127(5), 599-617. doi:10.1037/00332909.127.5.599 
ATTENTIONAL BIAS TO PAIN-RELATED INFORMATION

Posner, M. I. (1980). Orienting of attention. Quarterly Journal of Experimental Psychology, 32(1), 3-25. doi:10.1080/00335558008248231

Roelofs, J., Peters, M. L., Zeegers, M. P. A., \& Vlaeyen, J. W. S. (2002). The modified Stroop paradigm as a measure of selective attention towards pain-related stimuli among chronic pain patients: a meta-analysis. European Journal of Pain, 6(4), 273281. doi:10.1053/eujp.2002.0337

Schoth, D. E., \& Liossi, C. (2010). Attentional bias toward pictorial representations of pain in individuals with chronic headache. The Clinical Journal of Pain, 26(3), 244-250. doi:10.1097/AJP.0b013e3181bed0f9

Schoth, D. E., Nunes, V. D., \& Liossi, C. (2012). Attentional bias towards pain-related information in chronic pain: A meta-analysis of visual-probe investigations. Clinical Psychology Review, 32(1), 13-25. doi:10.1016/j.cpr.2011.09.004

Sharp, T. J. (2001). Chronic pain: A reformulation of the cognitive-behavioural model. Behaviour Research and Therapy, 39(7), 787-800. doi:10.1016/S00057967(00)00061-9

Sharpe, L., Brookes, M., Jones, E., Gittins, C., Wufong, E., \& Nicholas, M. (2017). Threat and fear of pain induces attentional bias to pain words: An eye $\square$ tracking study. European Journal of Pain, 21(2), 385-396. doi:10.1002/ejp.936

Sharpe, L., Haggman, S., Nicholas, M., Dear, B. F., \& Refshauge, K. (2014). Avoidance of affective pain stimuli predicts chronicity in patients with acute low back pain. Pain, 155(1), 45-52. doi:10.1016/j.pain.2013.09.004

Sharpe, L., Ianiello, M., Dear, B. F., Nicholson Perry, K., Refshauge, K., \& Nicholas, M. K. (2012). Is there a potential role for attention bias modification in pain patients? Results of 2 randomised, controlled trials. Pain, 153(3), 722-731. doi:10.1016/j.pain.2011.12.014 
ATTENTIONAL BIAS TO PAIN-RELATED INFORMATION

Sharpe, L., Johnson, A., \& Dear, B. (2015). Attention bias modification and its impact on experimental pain outcomes: Comparison of training with words versus faces in pain. European Journal of Pain, 19(9), 1248-1257. doi:10.1002/ejp.648

Sharpe, L., Nicholson Perry, K., Rogers, P., Dear, B. F., Nicholas, M. K., \& Refshauge, K. (2010). A comparison of the effect of attention training and relaxation on responses to pain. Pain, 150(3), 469-476. doi:10.1016/j.pain.2010.05.027

Sharpe, L., Nicholson Perry, K., Rogers, P., Refshauge, K., \& Nicholas, M. K. (2013). A comparison of the effect of mindfulness and relaxation on responses to acute experimental pain. European Journal of Pain, 17(5), 742-752. doi:10.1002/j.15322149.2012.00241.x

Stroop, J. R. (1935). Studies of interference in serial verbal reactions. Journal of Experimental Psychology, 18(6), 643-662. doi:10.1037/h0054651

Theeuwes, J. (2010). Top-down and bottom-up control of visual selection. Acta Psychologica, 135(2), 77-99. doi:10.1016/j.actpsy.2010.02.006

Todd, J., Sharpe, L., \& Colagiuri, B. (2016). Attentional bias modification and pain: The role of sensory and affective stimuli. Behaviour Research and Therapy, 83, 53-61. doi:10.1016/j.brat.2016.06.002

Todd, J., Sharpe, L., Johnson, A., Perry, K. N., Colagiuri, B., \& Dear, B. (2015). Towards a new model of attentional biases in the development, maintenance and management of pain. Pain, 156(9), 1589-1600. doi:10.1097/j.pain.0000000000000214

Van Bockstaele, B., Verschuere, B., Tibboel, H., De Houwer, J., Crombez, G., \& Koster, E. H. (2014). A review of current evidence for the causal impact of attentional bias on fear and anxiety. Psychological Bulletin, 140(3), 682-721. doi:10.1037/a0034834

Van Damme, S., Bulcke, C. V., Van Den Berghe, L., Poppe, L., \& Crombez, G. (2018). Do patients with chronic unilateral orofacial pain due to a temporomandibular disorder 
ATTENTIONAL BIAS TO PAIN-RELATED INFORMATION

show increased attending to somatosensory input at the painful side of the jaw? PeerJ, 6, e4310. doi:doi.org/10.7717/peerj.4310

Van Damme, S., Legrain, V., Vogt, J., \& Crombez, G. (2010). Keeping pain in mind: A motivational account of attention to pain. Neuroscience \& Biobehavioral Reviews, 34(2), 204-213. doi:10.1016/j.neubiorev.2009.01.005

Van Damme, S., Lorenz, J., Eccleston, C., Koster, E. H. W., De Clercq, A., \& Crombez, G. (2004). Fear-conditioned cues of impending pain facilitate attentional engagement. Clinical Neurophysiology, 34(1), 33-39. doi:10.1016/j.neucli.2003.11.001

Van Heck, C. H., Oosterman, J. M., de Kleijn, K., Jongsma, M. L., \& Van Rijn, C. M. (2017). Evidence for a priori existence of attentional bias subgroups in emotional processing of aversive stimuli. Frontiers in Behavioral Neuroscience, 11(87), 1-14. doi:10.3389/fnbeh.2017.00087

Van Ryckeghem, D., \& Crombez, G. (2014). Attentional bias and chronic pain: Where to go from here? Pain, 155, 6-7. doi:10.1016/j.pain.2013.10.25

Van Ryckeghem, D. M., \& Crombez, G. (2018). Pain and attention: Towards a motivational account. In P. Karoly \& G. Crombez (Eds.), Motivational Perspectives on Chronic Pain: Theory, Research, and Practice. New York: Oxford University Press.

Van Ryckeghem, D. M., \& Vervoort, T. (2016). Towards an integrative view of cognitive biases in pain. European Journal of Pain, 20(8), 1201-1202.

Van Ryckeghem, D. M. L., Crombez, G., Van Hulle, L., \& Van Damme, S. (2012). Attentional bias towards pain-related information diminishes the efficacy of distraction. Pain, 153(12), 2345-2351. doi:10.1016/j.pain.2012.07.032

Vervoort, T., Caes, L., Crombez, G., Koster, E., Van Damme, S., Dewitte, M., \& Goubert, L. (2011). Parental catastrophizing about children's pain and selective attention to 
ATTENTIONAL BIAS TO PAIN-RELATED INFORMATION

varying levels of facial expression of pain in children: A dot-probe study. Pain, 152(8), 1751-1757. doi:10.1016/j.pain.2011.03.015

Vlaeyen, J. W. S., \& Linton, S. J. (2000). Fear-avoidance and its consequences in chronic musculoskeletal pain: A state of the art. Pain, 85(3), 317-332. doi:10.1016/S03043959(99)00242-0

Vlaeyen, J. W. S., Morley, S., \& Crombez, G. (2016). The experimental analysis of the interruptive, interfering, and identity-distorting effects of chronic pain. Behaviour Research and Therapy, 86, 23-34. doi:10.1016/j.brat.2016.08.016

Vogt, J., De Houwer, J., Moors, A., Van Damme, S., \& Crombez, G. (2010). The automatic orienting of attention to goal-relevant stimuli. Acta Psychologica, 134(1), 61-69. doi:10.1016/j.actpsy.2009.12.006

Vos, T., Flaxman, A. D., Naghavi, M., Lozano, R., Michaud, C., Ezzati, M., Shibuya, K., Salomon, J. A., Abdalla, S., \& Aboyans, V. (2012). Years lived with disability (YLDs) for 1160 sequelae of 289 diseases and injuries 1990-2010: A systematic analysis for the Global Burden of Disease Study 2010. The Lancet, 380(9859), 21632196. doi:10.1016/S0140-6736(12)61729-2

Yang, Z., Jackson, T., \& Chen, H. (2013). Effects of chronic pain and pain-related fear on orienting and maintenance of attention: An eye movement study. The Journal of Pain, 14(10), 1148-1157. doi:10.1016/j.jpain.2013.04.017

Yang, Z., Jackson, T., Gao, X., \& Chen, H. (2012). Identifying selective visual attention biases related to fear of pain by tracking eye movements within a dot-probe paradigm. Pain, 153(8), 1742-1748. doi:10.1016/j.pain.2012.05.011 


\title{
Author Disclosures
}

\author{
Contributors
}

Authors A and B contributed equally to the manuscript and are therefore both first authors. All authors designed the study and contributed to the protocol. Authors A and B conducted the literature search and data extraction. Authors A and B contributed to the statistical analyses. The final meta-analyses were performed by author A. Author A wrote the first draft of the manuscript and all authors contributed to and have approved the final manuscript.

\section{Funding Sources}

Author B receives grant funding from the European Union's Horizon 2020 research and innovation program under the Marie Sklodowska-Curie grant agreement $N^{\circ} 706475$. The funding body had no role in the study design, collection, analysis or interpretation of the data, writing the manuscript, or the decision to submit the paper for publication. The other authors have no sources of funding to declare.

\section{Conflicts of Interest}

All authors declare that they have no conflicts of interest. 


\section{Acknowledgements}

The authors wish to thank the following authors of the manuscripts included in this meta-analysis for providing data files and further information about their studies: Gordon Asmundson, Marie Heloise Bardel, Corinna Baum, Andrew Bayliss, Jennifer Bowler, Mel Brookes, Blake Dear, Violeta Dimova, Samantha Fashler, Patrick Finan, Katarina Forkmann, Zoe Franklin, Eric Garland, Casper van Heck, Paul Holmes, Todd Jackson, Joel Katz, Ali Khatibi, Stefan Lautenbacher, Christina Liossi, Fei Lou, Michael McDermott, Somayeh Mohammadi, Tineke van Rijn, Jeffrey Roelofs, Jennifer Scheel, Daniel Schoth, Todd Smitherman, Tine Vervoort, JinYan Wang, Tim Woodman, Zhou Yang. The authors also wish to thank Nele Decoene for her assistance with article screening. 


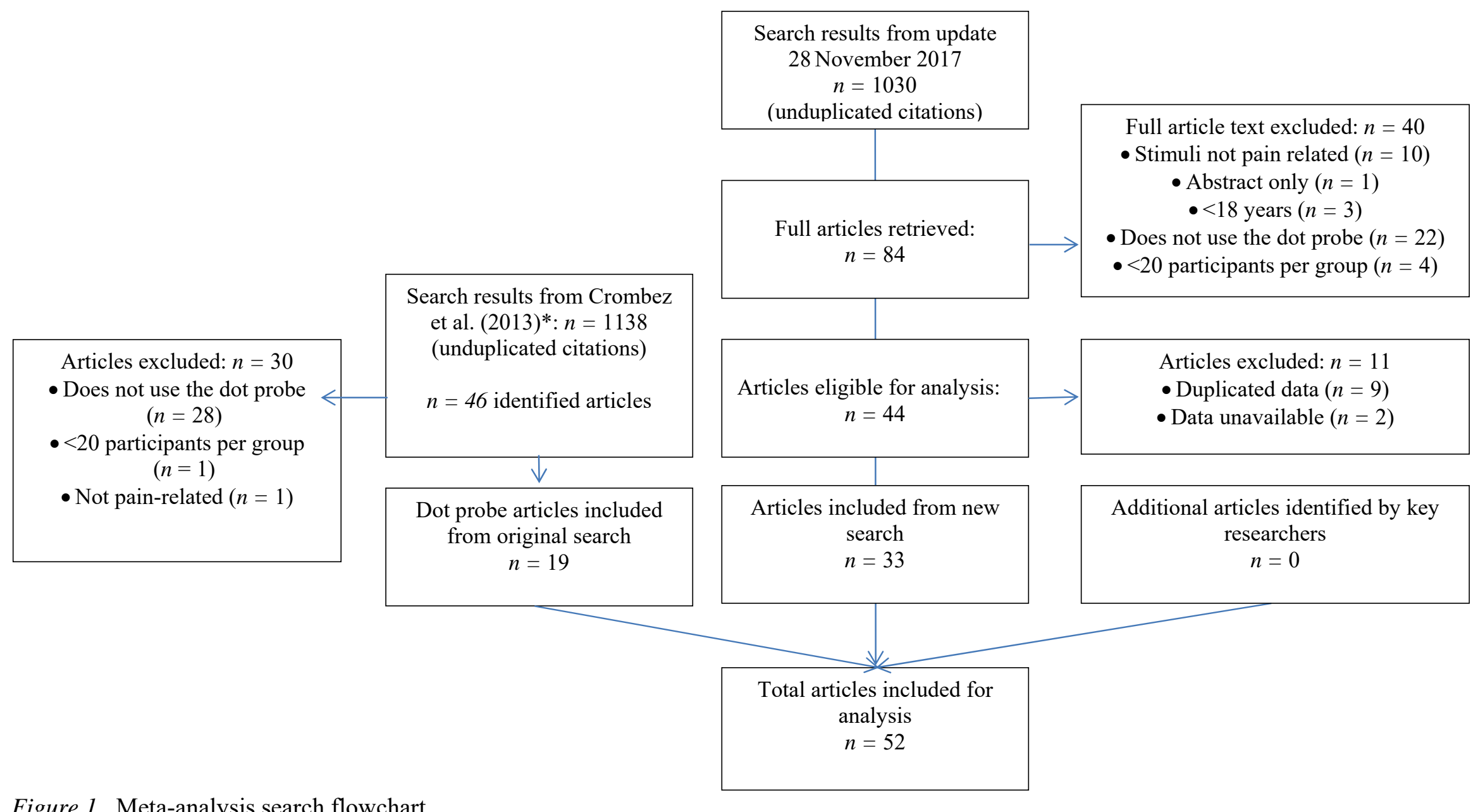

Figure 1. Meta-analysis search flowchart.

Note: *For full initial search strategy, see Crombez et al. (2013). 
Table 1

Comparing attentional biases across pain groups for pain words and pain pictures

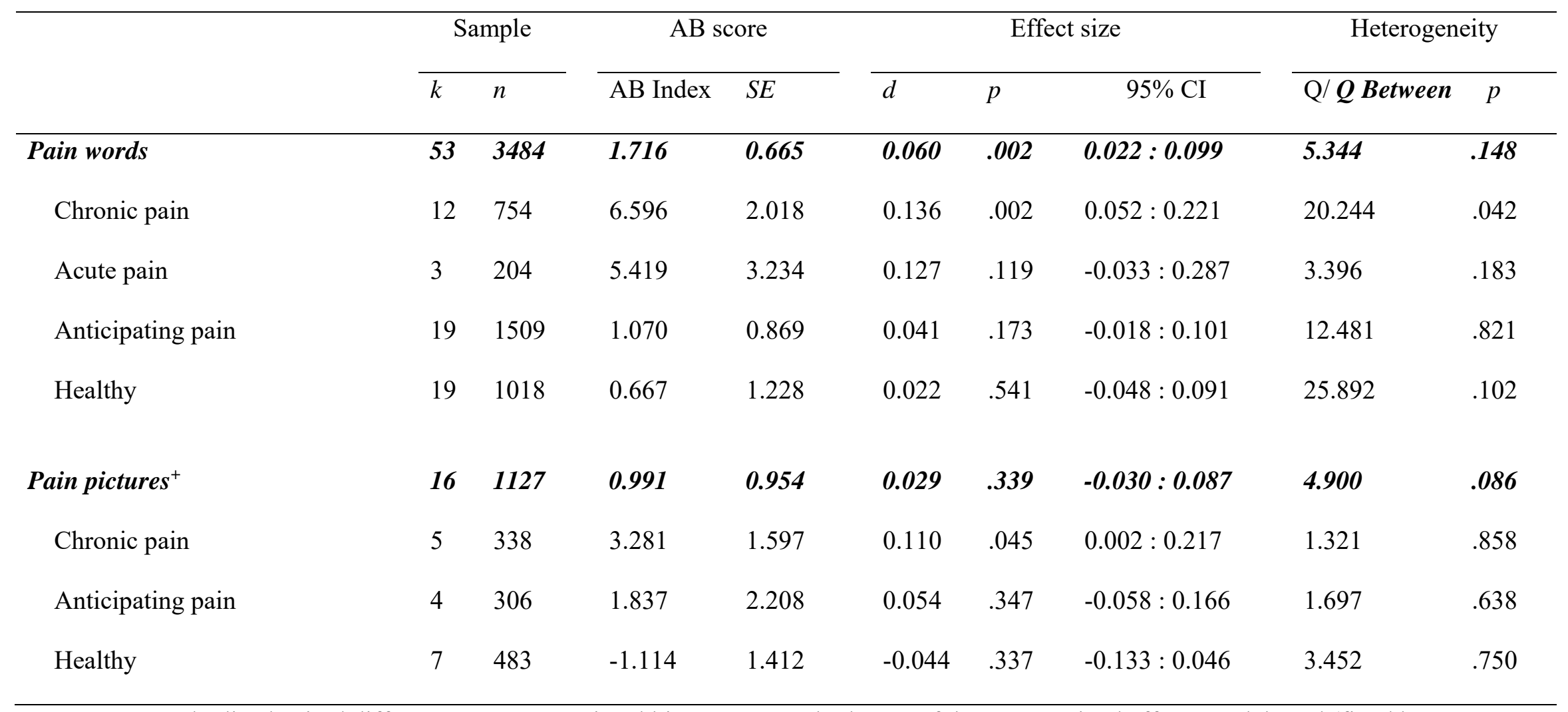

Notes: $d=$ standardized paired difference, $\mathrm{AB}=$ attentional bias, $S E=$ standard error of the mean. Mixed effects model used (fixed between studies, random between pain groups); where more than one value used in the study, outcomes averaged. ${ }^{+}$No studies used pain pictures within an acute pain sample. 
Table 1

Comparing attentional biases across pain groups for each type of word stimulus

\begin{tabular}{|c|c|c|c|c|c|c|c|c|c|}
\hline & \multicolumn{2}{|c|}{ Sample } & \multicolumn{2}{|c|}{ AB score } & \multicolumn{3}{|c|}{ Effect size } & \multicolumn{2}{|c|}{ Heterogeneity } \\
\hline Chronic pain & 12 & 759 & 9.143 & 2.068 & 0.198 & $<.001$ & $0.092: 0.304$ & 36.282 & $<.001$ \\
\hline Acute pain & 3 & 204 & 13.901 & 3.764 & 0.303 & .003 & $0.101: 0.504$ & 12.596 & .002 \\
\hline Anticipating pain & 18 & 1429 & 1.321 & 1.409 & 0.038 & .339 & $-0.040: 0.117$ & 16.221 & .508 \\
\hline Healthy & 17 & 949 & 0.445 & 1.443 & 0.014 & .760 & $-0.075: 0.103$ & 24.965 & .070 \\
\hline Affective pain words & 27 & 2037 & 0.942 & 0.786 & 0.029 & 190 & $-0.014: 0.073$ & 1.771 & .621 \\
\hline Anticipating pain & 10 & 939 & 0.977 & 1.057 & 0.037 & .259 & $-0.027: 0.101$ & 7.829 & .551 \\
\hline Healthy & 5 & 262 & 0.151 & 2.051 & 0.012 & .841 & $-0.109: 0.134$ & 1.880 & .758 \\
\hline
\end{tabular}




\begin{tabular}{|c|c|c|c|c|c|c|c|c|c|}
\hline Threat words & 32 & 2323 & -0.617 & 1.316 & -0.012 & .746 & $-0.081: 0.058$ & 6.088 & .107 \\
\hline Anticipating pain & 9 & 756 & -0.055 & 2.516 & -0.006 & .924 & $-0.134: 0.121$ & 10.875 & .209 \\
\hline Healthy & 12 & 745 & 2.320 & 2.023 & 0.082 & .167 & $-0.034: 0.199$ & 23.664 & .014 \\
\hline Health words & 28 & 1809 & -0.774 & 0.869 & -0.020 & .404 & $-0.066: 0.027$ & 0.422 & .936 \\
\hline Chronic pain & 9 & 637 & -1.302 & 1.651 & -0.023 & .558 & $-0.101: 0.055$ & 4.135 & .845 \\
\hline Acute pain & 3 & 205 & -2.159 & 2.876 & -0.051 & .462 & $-0.188: 0.086$ & 0.076 & .963 \\
\hline Positive words $^{+}$ & 13 & 932 & 0.185 & 1.022 & 0.004 & .897 & $-0.060: 0.069$ & 0.021 & .886 \\
\hline Anticipating pain & 7 & 490 & 0.493 & 1.787 & 0.009 & .847 & $-0.080: 0.097$ & 3.472 & .748 \\
\hline Healthy & 6 & 442 & 0.035 & 1.246 & -0.001 & .988 & $-0.094: 0.093$ & 0.702 & .983 \\
\hline
\end{tabular}

Notes: $d=$ standardized paired difference, $\mathrm{AB}=$ attentional bias, $S E=$ standard error of the mean. Mixed effects model used (fixed between studies, random between pain groups); where more than one value used in the study, outcomes averaged. ${ }^{+}$Chronic pain and acute pain groups excluded due to a lack of studies in these categories. 
Table 3

Attentional biases towards sensory pain words for chronic pain patients and healthy individuals, across parameters

\begin{tabular}{|c|c|c|c|c|c|c|c|c|c|c|c|c|}
\hline & \multicolumn{5}{|c|}{ Chronic pain patients } & \multicolumn{5}{|c|}{ Healthy individuals } & \multicolumn{2}{|c|}{ Comparison* } \\
\hline & \multicolumn{2}{|c|}{ Sample } & \multicolumn{3}{|c|}{ Effect size } & \multicolumn{2}{|c|}{ Sample } & \multicolumn{3}{|c|}{ Effect size } & \multirow[b]{2}{*}{$Q$} & \multirow[b]{2}{*}{$p$} \\
\hline & $k$ & $n$ & $d$ & $p$ & $95 \% \mathrm{CI}$ & $k$ & $n$ & $d$ & $p$ & $95 \% \mathrm{CI}$ & & \\
\hline Overall effect & 12 & 759 & 0.196 & .006 & $0.056: 0.336$ & 17 & 949 & 0.014 & .734 & $-0.068: 0.097$ & 4.798 & .028 \\
\hline \multicolumn{13}{|l|}{ Stimulus orientation } \\
\hline Upper-lower & 10 & 684 & 0.237 & .002 & $0.088: 0.387$ & 12 & 712 & -0.015 & .713 & $-0.098: 0.067$ & 8.462 & .004 \\
\hline Left-right & 2 & 75 & -0.060 & .603 & $-0.287: 0.167$ & 5 & 237 & 0.123 & .245 & $-0.084: 0.330$ & - & - \\
\hline \multicolumn{13}{|c|}{ Stimulus presentation time } \\
\hline Subliminal & - & - & - & - & - & 2 & 150 & -0.059 & .470 & $-0.219: 0.101$ & - & - \\
\hline$<500 \mathrm{~ms}$ & - & - & - & - & - & 2 & 150 & -0.097 & .594 & $-0.456: 0.261$ & - & - \\
\hline $500-1000 \mathrm{~ms}$ & 10 & 684 & 0.212 & .003 & $0.073: 0.350$ & 11 & 632 & 0.031 & .455 & $-0.050: 0.111$ & 4.901 & .027 \\
\hline$>1000 \mathrm{~ms}$ & 3 & 115 & 0.270 & .387 & $-0.341: 0.882$ & 6 & 277 & 0.045 & .715 & $-0.198: 0.289$ & 0.448 & .503 \\
\hline \multicolumn{13}{|l|}{ Task instructions } \\
\hline Process one stimulus & 2 & 66 & 0.087 & .483 & $-0.155: 0.329$ & 2 & 51 & -0.215 & .131 & $-0.494: 0.064$ & - & - \\
\hline Process both stimuli & 8 & 602 & 0.219 & .010 & $0.053: 0.384$ & 9 & 546 & 0.084 & .121 & $-0.022: 0.190$ & 1.806 & .179 \\
\hline
\end{tabular}




\begin{tabular}{|c|c|c|c|c|c|c|c|c|c|c|c|c|}
\hline No instructions & 2 & 91 & 0.214 & .533 & $-0.459: 0.888$ & 6 & 352 & -0.049 & .411 & $-0.164: 0.067$ & - & - \\
\hline \multicolumn{13}{|l|}{ Gaze maintenance } \\
\hline Absent & 10 & 684 & 0.237 & .002 & $0.088: 0.387$ & 13 & 782 & -0.007 & .866 & $-0.082: 0.069$ & 8.168 & .004 \\
\hline Present & 2 & 75 & -0.060 & .603 & $-0.287: 0.167$ & 4 & 167 & 0.145 & .317 & $-0.139: 0.429$ & - & - \\
\hline \multicolumn{13}{|l|}{ Target action } \\
\hline Probe discrimination & 6 & 547 & 0.239 & .019 & $0.039: 0.439$ & 4 & 233 & 0.053 & .491 & $-0.098: 0.204$ & 2.103 & .147 \\
\hline Probe localization & 4 & 146 & 0.170 & .275 & $-0.135: 0.474$ & 11 & 665 & 0.022 & .687 & $-0.082: 0.125$ & 0.811 & .368 \\
\hline Probe detection & 2 & 66 & 0.087 & .483 & $-0.155: 0.329$ & 2 & 51 & -0.215 & 0.131 & $-0.494: 0.064$ & - & - \\
\hline \multicolumn{13}{|l|}{ Response tool } \\
\hline Keyboard & 11 & 719 & 0.167 & .020 & $0.026: 0.309$ & 12 & 604 & -0.011 & .849 & $-0.121: 0.100$ & 3.795 & .051 \\
\hline Response box & 1 & 40 & 0.566 & .001 & $0.225: 0.906$ & 5 & 345 & 0.073 & .179 & $-0.033: 0.179$ & - & - \\
\hline \multicolumn{13}{|l|}{ Method of response } \\
\hline One hand & 2 & 66 & 0.087 & .483 & $-0.155: 0.329$ & 3 & 78 & -0.111 & .385 & $-0.360: 0.139$ & - & - \\
\hline Two hands & 10 & 693 & 0.214 & .009 & $0.054: 0.374$ & 12 & 718 & 0.045 & .347 & $-0.049: 0.138$ & 3.214 & .073 \\
\hline No specified & - & - & - & - & - & 2 & 153 & -0.047 & .749 & $-0.333: 0.240$ & - & - \\
\hline
\end{tabular}

Note: * comparisons only made where $\mathrm{k}>2$ for both chronic pain group and healthy group 